\title{
Simulation of Energy-Range Relation for Protons Impacting on N-4 Nuclear Emulsion
}

\author{
Weiping Wen ${ }^{\mathrm{a}}$, Guangwu $\mathrm{Li}^{\mathrm{b}}$ \\ National Key Laboratory for Science and Technology on Nuclear Data, China Institute of Atomic \\ Energy, Beijing, 102413, China \\ aping.wwei@163.com, bowli@ciae.ac.cn
}

\begin{abstract}
Keywords:Monte Carlo, Nuclear Emulsion, Energy-Range Relation, Curve Fitting
Abstract:Therelationship between the energy of a high-speed proton when impacting onthe N-4 nuclear emulsion plane perpendicularlyand its range in the emulsion was investigated using classical SRIMMonte Carlo programand curve fitting method. A definite analytic expression was derived from this simulation with satisfactory residuals. This resulthas its significance for the prediction of experiments.
\end{abstract}

\section{Introduction}

Nuclear emulsionis made of a gel with interspersed $\mathrm{AgBr}$ crystals. It has been amongthe earliestparticle detectors used in high-energy physics and has inherent advantages when detecting neutrons in high energy[1]. Neutrons can be detected via recoil proton tracks in the nuclear emulsion and the neutron energy spectrum can then be determined from length distribution of the tracks $[2,3]$. To determine the energy of each incident neutron with respect to the proton recoil trajectory, the usual procedure is to accept proton recoils within a given angular criteria, measure the projection in the plane of the emulsion of the ranges of the recoil protons, and make an average correction to the deduced neutron energies. When using the nuclear recoil method to detect neutrons, most of the energy of the neutrons are passed to the recoil protons.Especially when the incident neutronsimpact on thenuclear emulsion planeperpendicularly, energy of the recoil protons roughly equal to that of the incident neutrons. Therefore, the energy of the incident neutrons will be able to be calculated quickly if the relationship between the energy and range of the recoil proton in the nuclear emulsion has been known. Here, we use the Monte Carlo program SRIM to simulate the energy loss of the recoil protons. And the method of curve fitting is adopted to establish the relationship of the energy and range of the protons.

\section{The simulation principle}

The nuclear emulsion consists of the following components: Silver halides, Gelatin and glycerin, Water and Nuclei of CNO group.The glycerin works as plasticizer for preventing nuclear emulsion stack from breaking. It may be pointed out that about $71 \%$ of interactions occur with heavy emulsion nuclei(AgBr), approximately 25\% due to light emulsion nuclei, i.e. Carbon, Nitrogen and Oxygen, and only 4\% with hydrogen nuclei. The number of the main atoms per unit volume of the nuclear emulsion is shown in Table 1.

Table 1 Main elements in the nuclear emulsion

\begin{tabular}{c|cccccccc}
\hline Element & $\mathrm{H}$ & $\mathrm{C}$ & $\mathrm{N}$ & $\mathrm{O}$ & $\mathrm{S}$ & $\mathrm{Br}$ & $\mathrm{Ag}$ & $\mathrm{I}$ \\
\hline $\begin{array}{c}\text { Number } \\
\left(\times 10^{22} \text { atoms/c.c }\right)\end{array}$ & 3.63 & 1.81 & 0.336 & 0.966 & 0.0147 & 0.0867 & 0.887 & 0.027 \\
\hline
\end{tabular}

When a proton passes through nuclear emulsion, it loses its energy by electromagnetic interactions. The energy lost by the proton is transferred to the electrons of the atoms. As a result of this, the later goes to an excited state. If the energy transferred to the electron is greater than the ionization potential of the atom, the electron is free to move from atom and the atom is said to be ionized. It may be mentioned that the most important mode of energy loss of a charged particle is the ionization [4]. The ionization of the atom converts some of the silver halide grains in such a way that they when immersed in a reducing bath, known as developer, get converted into silver grains. 
Therefore as the charged particle passes, grains are formed, showing the path of the particle, which is termed as the track of the particle. So the range of a recoil proton is the average distance traversed by a particle in unprocessed emulsion before its kinetic energy reduces to zero. The characteristics of the track depend on the nature and velocity of the charged particle. Higher the velocity of the charged particle, rarer will be the grain formed by it and vice versa.

The ionization energy loss of the charged particle having charge ze, velocity $v$ and mass $m$ when traversing through nuclear emulsioncan be described as the formula (1) [5]:

$$
-\frac{\mathrm{dE}}{\mathrm{dx}}=\frac{4 \pi z^{2} e^{4} N}{m v^{2}}\left(\frac{\sum_{i=1}^{8} \rho_{i} Z_{i}}{A_{i}}\right)\left[\ln \left(\frac{2 m v^{2}}{\tilde{I}\left(1-\beta^{2}\right)}\right)-\beta^{2}\right]
$$

Where $\tilde{I}$ is the average ionization potential, $\rho_{i}, Z_{i}$ and $A_{i}$ are the density, charge and atom number of the main elements in the emulsion, respectively. $\beta=v / c, c$ is the speed of light, $N$ is the number of the atoms per unit volume of the target.

\section{The simulation result}

SRIM is a MonteCarlo calculation which follows the ion into the target, making detailed calculations of the energy transferred to every target atom collision. Position of the particle, the energy loss of secondary particles and various parameters are stored and tracked throughout the whole process. Finally the expected values and the corresponding statistical errors of various physical quantities required are obtained. It always refers to the moving atom as an "ion", and all target atoms as "atoms". The impact of the incident ions and target nuclei is described by two-body collision theory. The distance as well as parameters after collision are obtained by random sampling. The incident energy of the proton ranges from $1 \mathrm{keV}$ to $14 \mathrm{MeV}$ in this simulation. We define a complex emulsion target made of compound materials including the above-mentioned eight different elements. The density of the emulsion is $3.4 \mathrm{~g} / \mathrm{cm}^{3}$. The incident angle of the ion with respect to the target surface is defined to be $90^{\circ}$, along the X-axis at an angle defined as $0^{\circ}$. After all the information are input, the simulation may start. We will find the output file after finishing the calculation as the followingfigure 1 .

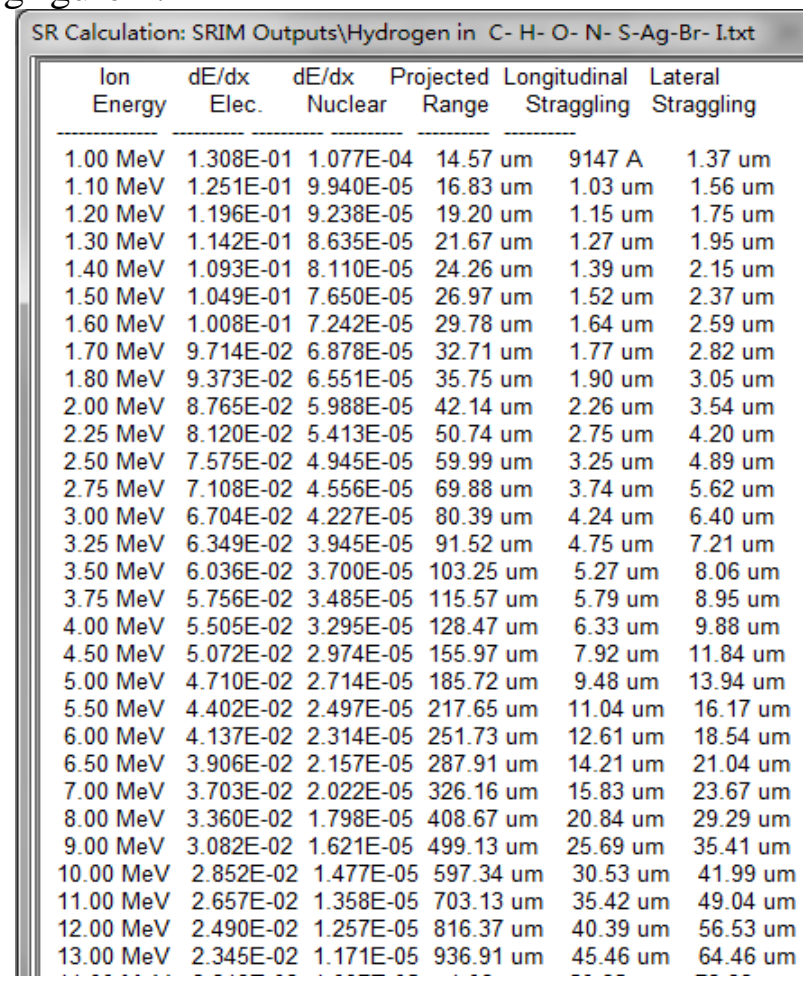

Fig.1Part of the simulation results

In order to calculate the recoil proton energy accurately, we must have the fit function relation between the energy and range of the protons, which need to meet the requirements of smooth curve 
and high accuracy. Because the proton energy is high, the argument value of the function will be large whose high-order terms will lead to great impact on the calculation results. In order the function value not to deviate too much from the experiment point, 3 time fitting curve equation will be adopted. 83 data points have been selected from the simulation results that covering the energy range from $10 \mathrm{keV}$ to $14 \mathrm{MeV}$ to be used for analysis. The cubic polynomial is expressed as formula (2).

$$
R_{p}=a x^{3}+b x^{2}+c x+d
$$

Where $R_{p}$ (um)and $x(\mathrm{MeV})$ are the range and energy of the recoil protons respectively. The parameters of the fitting function are shown in Table 2. The curve of fitting function and the residual plot of fitting results are shown in Fig.2 and Fig.3 respectively.

Table 2 Coefficients of fitting function

\begin{tabular}{c|cccc}
\hline Parameter & $\begin{array}{c}\boldsymbol{a} \\
\left(\mathrm{um} / \mathrm{MeV}^{3}\right)\end{array}$ & $\begin{array}{c}\boldsymbol{b} \\
\left(\mathrm{um} / \mathrm{MeV}^{2}\right)\end{array}$ & $\begin{array}{c}\boldsymbol{c} \\
(\mathrm{um} / \mathrm{MeV})\end{array}$ & $\begin{array}{c}\boldsymbol{d} \\
\text { (um) }\end{array}$ \\
\hline Value & -0.071 & 5.653 & 10.524 & -0.521 \\
Error (\%) & 0.18 & 3.38 & 15.09 & 10.70 \\
\hline
\end{tabular}

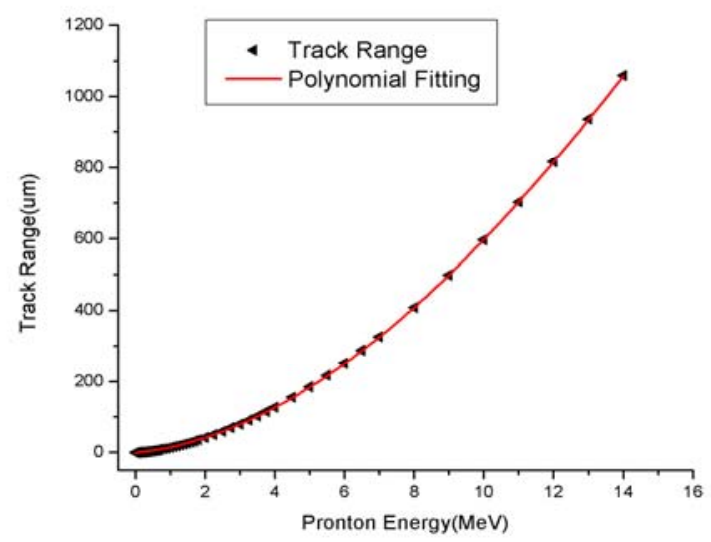

Fig. 2Energy-range relation of the recoil proton

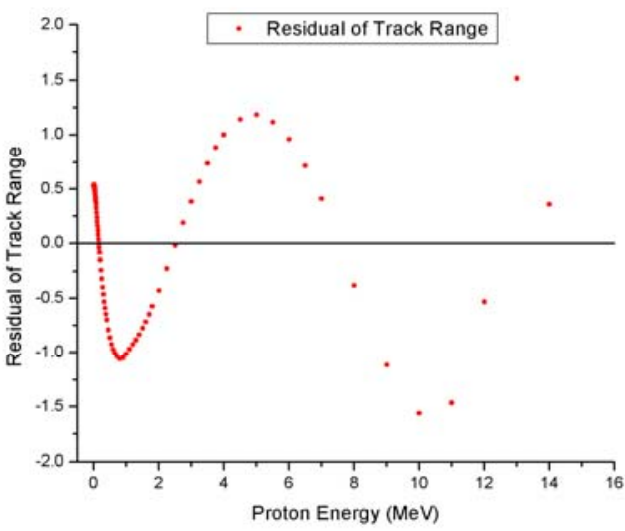

Fig.3Residual plot of the fitting results

Fig.3shows that the residuals of the fitting results are between the range $(-2,2)$ and this satisfies the need of accuracy.

\section{Conclusions}

Monte Carlo simulation and curve fitting method are used to establish therelationship between the energy and range of the recoil protonin nuclear emulsion in this paper. This relationship has universality because the data about the nuclear emulsion such as the density and constituents are provided by supplier on the basis of numerousmeasurement results. These results show that we can accurately estimate the energies of the protons impacting on the nuclear emulsion for the reason that the Monte Carlo simulation is an accumulation of a large number of random events. Besides, a fact is to be stressed that the final simulation distributions will scale with emulsion density, so any discrepancy in density will directly transcribe into differences in ranges.

\section{Acknowledgements}

It is a project supported by the National Natural Science Foundation of China (11275277). 


\section{References}

[1]Hancheng Sun, Donghai Zhang, Nuclear emulsion and high-energy physics, Radiation Measurements, 43 (2008): S139-S143.

[2] L. Cranberg, G. Rrye, N. Nereson and L. Rosen, Fission neutron spectrum of ${ }^{235}$,Physical Review, 103(1956): 662-670.

[3] A.B.Smith, R. K. Sjoblom and J. H. Roberts, Prompt fission neutron spectrum of $\mathrm{Pu}^{241}$, Physical Review, 123(1961): 2140-2142.

[4]J. F. Ziegler, J. P. Biersack, U. Littmark, The stopping and range of ions in solids. New York: Pergamon Press,1985:14-141.

[5] J. F. Ziegler and J. M. Manoyan, The stopping of ions in compounds, Nuclear Instruments andMethods, B35 (1989):215-228. 